\title{
Sharing Our Experience of Operating an Endoscopy Unit in the Midst of a COVID-19 Outbreak
}

\author{
Jimin Han and Eun Young Kim \\ Department of Internal Medicine, Daegu Catholic University School of Medicine, Daegu, Korea
}

On March 11, 2020, the World Health Organization (WHO) has officially called the Coronavirus disease 2019 (COVID-19) a pandemic. Now, cases of COVID-19 are being reported from all over the globe and anyone could be affected. Since the first confirmed case (a Chinese visitor from Wuhan) in the Republic of Korea was reported on January 21, 2020, ${ }^{1}$ our endoscopy unit has been operated under the strict attention on safety in order to protect healthcare personnel as well as patients. In this brief report, we would like to share our experience of managing an endoscopy unit during a COVID-19 outbreak.

As of midnight on March 18, there were a total of 8,413 confirmed cases of COVID-19 in the Republic of Korea. Of these, 6,144 cases (73\%) of them were from Daegu, the city in which our hospital is located. Ever since the first case of COVID-19 in Daegu was reported on February 18, our hospital had implemented a rigorous rule of wearing facial masks (surgical mask or N95) and frequent handwashing for all healthcare personnel in the endoscopy unit. However, one of the nurses in the endoscopy unit was diagnosed with COVID-19 on February 27, 2020. It was not a hospital acquired infection but the exact route of infection is unknown. The endoscopy unit was then closed for two days for disinfection. After testing all the other nurses for COVID-19, self-isolation at home for two weeks were enforced. The nurses other than the infected

Received: March 23, 2020 Revised: March 24, 2020

Accepted: March 25, 2020

Correspondence: Eun Young Kim

Division of Gastroenterology, Department of Internal Medicine, Daegu Catholic University School of Medicine, 33 Duryugongwon-ro 17-gil, Nam-gu, Daegu 42472, Korea

Tel: +82-53-650-4211, Fax: +82-53-624-3281, E-mail: kimey@cu.ac.kr ORCID: https://orcid.org/0000-0003-3965-9964

(c) This is an Open Access article distributed under the terms of the Creative Commons Attribution Non-Commercial License (http://creativecommons.org/ licenses/by-nc/3.0) which permits unrestricted non-commercial use, distribution, and reproduction in any medium, provided the original work is properly cited. one returned to work after two negative consecutive tests for COVID-19. Every gastroenterologist working in the unit was also tested for COVID-19 and the results were all negative. This prevention of person-to-person transmission strongly highlights the importance of wearing facial masks and frequent handwashing to prevent spread of disease in the workplace.

When the endoscopy unit was reopened after being disinfected for two days, only a limited number of endoscopic procedures could be performed with the help of nurses from our health promotion center. Because an airborne infection isolation (negative pressure) room is not available in this endoscopy unit, the only one room with windows that can be opened after the procedure was utilized. Indications for endoscopic procedures were redefined to include urgent situations (e.g., gastrointestinal [GI] bleeding, acute cholangitis, foreign body, luminal obstruction) and care for cancer patients (e.g., biopsy, biliary and luminal stenting). Non-urgent procedures were rescheduled to a later date. Prior to endoscopic procedures, every patient was screened and risk stratified for COVID-19 by history taking for potential exposure to infection, symptoms such as fever and coughing, and measurement of body temperature. ${ }^{2,3} \mathrm{He} /$ she had to wear a facial mask and could take it off immediately before starting the actual endoscopic procedure. After an endoscopic procedure under conscious sedation, the patient could wear a facial mask again only if their $\mathrm{PaO}_{2}$ was above 95\% with room air. All endoscopic exams should be considered aerosol generating procedures ${ }^{3}$ and fecal viral RNAs have been detected in 53\% of patients with COVID-19. ${ }^{4}$ Therefore, all healthcare personnel were required to wear proper personal protection equipment (PPE) including N95 or KF94 masks, disposable plastic gowns, gloves, and eye protection with goggles or a facial shield.

The following is about performing colonoscopy in a pa- 
tient who came into close contact with a confirmed case of COVID-19. A 59-years old male with hematochezia was transferred to the emergency room (ER) from other hospital. His initial COVID-19 test was negative. Colonoscopy was planned to evaluate the cause of hematochezia. The patient was transported to the endoscopy unit from ER using preplanned route designed to minimize contact with other people in the hospital. All other healthcare personnel were kept in a designated place separate from the patient and three staff members required for sigmoidoscopy. These three included one endoscopist, one nurse, and one person for scope reprocessing. All these staff members wore hair caps, foot wraps, disposable plastic gowns over long-sleeved surgical gowns, and two layers of gloves (with one over the wrist of the plastic gown), goggles, and N95 facial masks for the procedure (Fig. 1). The exam was uneventful and the endoscopic findings were compatible with severe ulcerative colitis. After the procedure, the scope was reprocessed according to the Korean Society of

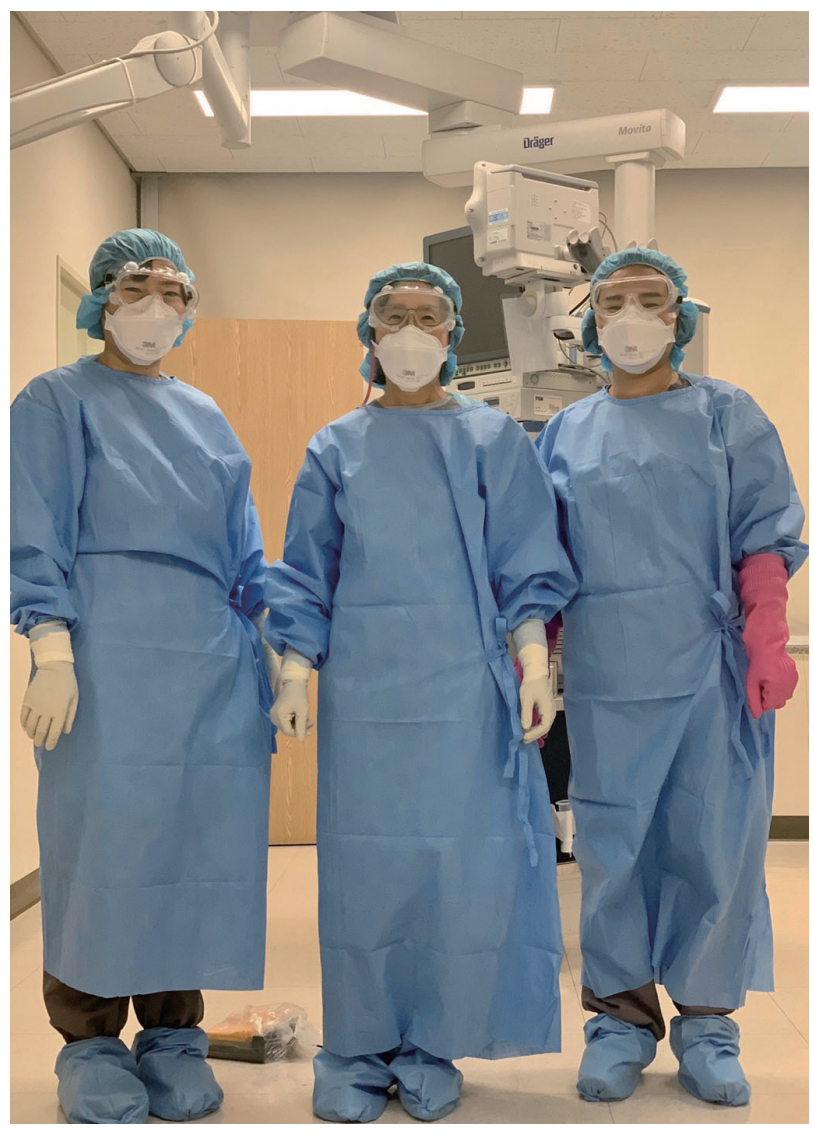

Fig. 1. Endoscopy unit staffs after donning personal protection equipment (from left to right, assisting nurse, endoscopist, and scope handler). All staffs are wearing hair caps, a pair of goggles, N95 facial masks, disposable gowns, two layers of gloves (one pair of non-latex exam gloves and another pair of latex surgical gloves or rubber gloves), and pairs of shoe covers. During the endoscopic exam, another disposable plastic gown was worn over the already donned gown.
Gastrointestinal Endoscopy guideline. ${ }^{5,6}$ The endoscopy room was cleaned including all exposed surfaces and the window of the room was kept opened for two hours. If an endoscopic procedure for a confirmed case of COVID-19 were to be done, level-D protective clothing is required for all the involved personnel.

During the two weeks of self-isolation of the endoscopy unit nurses, 20 procedures of urgent endoscopic retrograde cholangiopancreatography (ERCP) were performed. In addition to standard radiation protection, all the involved healthcare personnel wore PPE as described previously. Since radiation protective goggles are already put on, facial shield was worn over the goggles and N95 or KF94 facial mask. The greatest difficulty in performing ERCP was risk stratification, as $50 \%$ of the patients who required ERCP had fever. Presence of fever automatically caused the patient to be stratified as having an intermediate ${ }^{2}$ or high risk ${ }^{3}$ of potential infection. Because COVID-19 test took about one day at that time, some patients had to undergo ERCP before knowing the test results. Prior to deciding to perform ERCP in a febrile patient, the endoscopist reviewed chest X-ray and arterial blood gas analysis in addition to contact history, symptoms, and body temperature. When a patient was suspected to have respiratory tract infection, they underwent a computed tomography of the chest. If there was no cause for fever besides underlying pancreatobiliary disease, ERCP was performed. As of March 21,2020 , there has been no confirmed case of COVID-19 infection among the patients who underwent ERCP.

During the COVID-19 outbreak, both healthcare personnel and patients are at risk of infection via person-to-person contact, aerosol, and contact with virus-contaminated fluids. ${ }^{3}$ The symptoms of COVID-19 are known to be mainly respiratory symptom, but some patients may complain of GI symptoms. ${ }^{7}$ For example, an 85-year-old man came to our outpatient clinic complaining of abdominal pain, diarrhea, and general weakness. He did not have fever, cough, or dyspnea. One week later, he was confirmed to have COVID-19. The patient's GI doctor had not been infected despite prolonged close contact conducting history taking and physical examination in an exam room. Both, the gastroenterologists and patient wore a KF94 facial mask each respectively and handwashing was done immediately after physical contact with the patient. All the other personnel in the outpatient clinic wore KF94 facial masks. Such patients may visit outpatient clinic and will likely undergo endoscopic examinations due to their GI symptoms. Safety measures to protect healthcare personnel are therefore always needed in outpatient clinics given that the personnel can incidentally be exposed to COVID-19 patients.

GI endoscopy has the potential of spreading COVID-19 infection because of the following reasons: (1) the procedure 
requires close contact between healthcare personnel and patients, (2) cough, retching, and flatus generate aerosols, and (3) oral and/or fecal materials often contaminate their surroundings during GI endoscopy. To eliminate the risk of COVID-19 transmission in the endoscopy unit, rigorous safety measures for both healthcare personnel and patients are prerequisite. This pandemic, too, shall pass. Until then, the best strategy is frequent handwashing, proper use of PPE, and rigorous enforcement of reprocessing and disinfection of the endoscopic unit.

\section{Conflicts of Interest}

The authors have no financial conflicts of interest.

\section{Author Contributions}

Conceptualization: Eun Young Kim

Data curation: Jimin Han, EYK

Formal analysis: JH, EYK

Investigation: JH, EYK

Methodology: JH, EYK

Project administration: EYK

Resources: JH, EYK

Supervision: EYK

Validation: EYK

Visualization: EYK

Writing-original draft: JH, EYK

Writing-review\&editing: JH, EYK
ORCID

Jimin Han: https://orcid.org/0000-0001-8674-370X

\section{REFERENCES}

1. Kim JY, Choe PG, Oh Y, et al. The first case of 2019 novel coronavirus pneumonia imported into Korea from Wuhan, China: implication for infection prevention and control measures. J Korean Med Sci 2020;35:e61.

2. Repici A, Maselli R, Colombo M, et al. Coronavirus (COVID-19) outbreak: what the department of endoscopy should know. Gastrointest Endosc 2020 Mar 13 [Epub]. https://doi.org/10.1016/j.gie.2020.03.019.

3. Soetikno R, Teoh AY, Kaltenbach T, et al. Considerations in performing endoscopy during the COVID-19 pandemic. Gastrointest Endosc 2020 [In press]. https://www.epege.gr/wp-content/uploads/2020/03/Considerations-in-performing-endoscopy-during-the-COVID-19-pandemic-1. pdf.

4. Xiao F, Tang M, Zheng X, Liu Y, Li X, Shan H. Evidence for gastrointestinal infection of SARS-CoV-2. Gastroenterology 2020 Mar 3 [Epub]. https://doi.org/10.1053/j.gastro.2020.02.055.

5. Oh HJ, Kim JS. Clinical practice guidelines for endoscope reprocessing. Clin Endosc 2015;48:364-368.

6. Shin JE, Jung Y, Lee JH, et al. Updates on the disinfection and infection control process of the accredited endoscopy unit. Clin Endosc 2019;52:443-450.

7. Gu J, Han B, Wang J. COVID-19: gastrointestinal manifestations and potential fecal-oral transmission. Gastroenterology 2020 Mar 3 [Epub]. https://doi.org/10.1053/.j.gastro.2020.02.054. 